\title{
Avaliação do desempenho do acoplamento de sinais Power Line Communication em circuitos de média tensão típicos brasileiros
}

Luís F. Sauthier, Paulo S. Sausen, Maurício de Campos, Airam T. Z. R. Sausen ${ }^{1}$ DCEEng/UNIJUI, Ijuí, RS Alexandre C. Oliveira, Benedito A. Luciano ${ }^{2}$

DEE/UFCG, Campina Grande, PB

Rene R. Emmel Jr. ${ }^{3}$

DRS/CEEE-D, Porto Alegre, RS

Resumo. A tecnologia Power Line Communication (PLC) já está consolidada em vários países europeus, além de países como os EUA e Canadá. No entanto, o seu uso no Brasil ainda é bastante restrito, especialmente pelas particularidades das redes elétricas brasileiras. Nesse sentido, atualmente estudos dedicados e propositivos de metodologias de adaptação desta tecnologia para as características das redes brasileiras ainda são raros. Nesse contexto, o principal objetivo deste artigo é analisar estratégias de acoplamento de sinais PLC em circuitos de média tensão, em sistemas de distribuição de energia elétrica, através de acopladores indutivos e transformadores de potência, já presentes nos sistemas de distribuição. Um modelo computacional é proposto, e as simulações computacionais são realizadas a partir de um conjunto de dados obtidos de uma concessionária de energia do sul do Brasil objetivando analisar o real comportamento da tecnologia PLC em uma típica rede brasileira.

Palavras-chave. PLC, Acoplador Indutivo, Smart Grids, Média Tensão.

\section{Introdução}

O crescimento do consumo e da demanda de energia elétrica no mundo têm representado um desafio para o setor de energia. Essas taxas de crescimento significativas, causam preocupação e requerem investimentos para que o sistema continue a operar de forma segura e confiável. Em alguns estudos, como em [9], os autores demonstram que as redes de energia convencionais sofrem com a falta de análise automatizada, o tempo de resposta lento, o controle limitado e a má coordenação entre a geração e o consumo de energia, resultando em inúmeros problemas nas últimas décadas. Além disso, a interrupção no fornecimento de eletricidade afeta negativamente os consumidores com diferentes níveis de impacto, com base na natureza da atividade. Para enfrentar esses desafios é necessário introduzir ao sistema elétrico convencional algumas tecnologias, o que tem motivado o desenvolvimento das redes elétricas inteligentes (do inglês, Smart Grids).

Uma dessas tecnologias é a denominada Power Line Comunnication (PLC), que consiste em transmitir dados pela rede de energia elétrica. Com a demanda por aplicações de redes elétricas inteligentes, há um crescente interesse na comunicação utilizando a própria infraestrutura da rede

\footnotetext{
${ }^{1}$ luis.sauthier@unijui.edu.br, sausen@unijui.edu.br, campos@unijui.edu.br, airam@unijui.edu.br.

2 aco@dee.ufcg.edu.br, benedito.luciano@dee.ufcg.edu.br.

${ }^{3}$ ReneEJ@ceee.com.br.
} 
elétrica. Na Europa, por exemplo, o uso da tecnologia PLC tem se consolidado, contabilizando a instalação de mais de 30 milhões de dispositivos baseados em modems do padrão G3 (i.e., G3 Alliance) e mais de 20 milhões de medidores de energia baseados em modems do padrão PRIME (i.e., PRIME Alliance) [10]. Apesar de antiga, apenas na última década a tecnologia PLC superou a dificuldade de transmitir informações por meio da rede elétrica, a qual não foi idealmente projetada para esse fim.

No cenário brasileiro, as implementações da tecnologia PLC ainda são insipientes. Conforme apresentado em [11] estão relacionadas as aplicações de sistemas de medição inteligentes (do inglês, Smart Metering), em geral, restritas aos circuitos de Baixa Tensão (BT). A ampliação do uso da tecnologia PLC, para dar suporte a outras aplicações dos sistemas de distribuição, inclusive contemplando cenários onde existe a inserção de geração distribuída, exige que o confinamento do PLC à rede de BT seja superado, expandido o seu alcance às redes de Média Tensão (MT).

Neste contexto, o principal objetivo deste trabalho é fornecer subsídios para ampliar a discussão da aplicação da tecnologia PLC às redes elétricas de MT brasileiras. Essa proposta é apresentada a partir da realização da análise das estratégias de acoplamento de sinais PLC em circuitos de média tensão, de sistemas de distribuição de energia elétrica, através de acopladores indutivos e transformadores de potência já presentes nos sistemas de distribuição. Desta forma, utilizando um par de modems PLC operando na configuração do padrão G3, foi possível avaliar e analisar a conexão entre os modens. Além disso, por meio de ajuste de parâmetros foi possível obter um modelo computacional que representa o comportamento do transformador sob características de alta frequência.

O restante deste artigo está organizado da seguinte forma. Na Seção 2 é discutida a aplicação da tecnologia PLC na MT, na sequência são apresentadas as análises dos modelos de acopladores para sinais PLC na MT. Já na Seção 3 é apresentado o estudo de caso com as medições relativas ao estudo do comportamento do sinal PLC. E finalmente na Seção 4 são apresentadas as conclusões deste trabalho e as possibilidades de trabalhos futuros.

\section{Referencial teórico}

\subsection{PLC na média tensão}

A tecnologia PLC pode ser dividida em duas categorias: PLC Banda Estreita (NarrowBand PLC - NB PLC) e PLC Banda Larga (BroadBand PLC - BB PLC). Em geral a NB PLC é a tecnologia empregada na implantação dos sistemas de suporte às operações das concessionárias de energia, enquanto a BB PLC tem sido empregada para suporte a serviços de internet através da rede elétrica, o que não impede o seu uso, também para suporte a serviços privativos das concessionárias de energia. A NB PLC opera com taxa de dados algumas dezenas de kilobits e abaixo da frequência de $1 \mathrm{MHZ}$, nessa categoria, a normatização dos parâmetros de comunicação seguem um dos seguintes padrões CENELEC (CENELEC EN50065-1/A1), IEEE 1901.2a (IEEE 1901.2-2015) e ARIB (ARIB - Association of Radio Industries and Business).

Basicamente, os dados a serem transmitidos, após serem devidamente modulados, são injetados nos cabos elétricos por meio de um circuito de acoplamento. O sinal PLC resultante (dados modulados) pode coexistir com os $50 / 60 \mathrm{~Hz}$ da rede elétrica, operando em uma frequência significativamente mais alta. Na essência, o circuito de acoplamento é a única divergência entre um sistema PLC para BT e MT. Devido ao custo e tamanho, os componentes utilizados no circuito do acoplador do PLC são passivos (resistores, capacitores, transformadores e indutores).

Uma análise preliminar poderia suscitar a ideia de que o ambiente elétrico nos circuitos de MT pudessem apresentar menores níveis de ruído, visto que o acoplamento entre o circuito de MT e BT se dá através de um transformador de distribuição. Contudo, existe a possibilidade 
de níveis significativos de atenuação entre os circuitos primário e secundário dos transformadores, a propagação de sinais de alta frequência ocorre em ambos os sentidos, sendo mais favorecido o percurso da BT para a MT conforme apresentado em [12].

Além disso o percurso dos sinais de alta frequência são favorecidos no sentido da BT para a MT, através do transformador de distribuição, infere-se desta análise que há um maior nível de confinamento dos sinais no circuito de MT. Não obstante, haver a prevalência de transmissão de sinais da baixa para a média tensão, dado que a diferença de nível de atenuação em alguns casos pode ser pequena. Dependendo do modelo do transformador de distribuição, é possível considerálo como elemento acoplador. Portanto, pode-se considerar na implantação de um sistema PLC na MT as seguintes topologias:

- Injeção/recuperação de sinal através de acoplador PLC instalado na MT. O sinal pode ser injetado usando acopladores indutivos ou capacitivos;

- Injeção/recuperação de sinal no circuito de BT, usando o transformador de distribuição.

\subsection{Transformador de distribuição como elemento acoplador}

Alguns trabalhos envolvendo o estudo do uso do transformador como elemento acoplador como por exemplo em [8], demonstram que sua utilização apresenta desafios significativos, pois ele atua como um forte mecanismo de atenuação do sinal PLC (até 30-40 dB dependendo da frequência de operação do PLC). Na modelagem do transformador, ao analisar o comportamento do mesmo submetido a sinais de frequência variável, ou seja, a assinatura do transformador, demonstra o fato de os parâmetros do transformador ( $\mathrm{R}, \mathrm{L}$ e $\mathrm{C})$, quando submetidos a frequências elevadas, variam com a frequência e não podem ser considerados como parâmetros lineares.

Desta forma, ao introduzir um sinal de alta frequência no transformador, é necessário levar em consideração as indutâncias próprias das bobinas do primário e do secundário e as mútuas entre elas, o efeito pelicular (skin effect). Além das perdas por correntes parasitas e as capacitâncias própria das bobinas e mútuas entre elas, assim como o efeito do comprimento dos cabos nos lados de MT e de BT [5], conforme mencionado anteriormente.

Algumas dificuldades no estabelecimento de modelos representativos dos parâmetros dos transformadores monofásicos e trifásicos de forma exata dizem respeito às trajetórias dos fluxos magnéticos de dispersão, à anisotropia magnética e à não-linearidade do material do núcleo e à própria geometria de todas as partes que compõem esses equipamentos [7]. Entretanto, algumas dessas dificuldades têm sido mitigadas ou superadas por meio de trabalhos de pesquisa e desenvolvimento realizados desde o início do decênio de 1990 [1], assim como alguns trabalhos publicados recentemente, dentre os quais, pode-se citar [2].

\subsection{Acopladores PLC para média tensão}

Um estudo detalhado relativo à acopladores PLC em MT é apresentado em [3]. Considerando o critério de conexão física para classificação dos dispositivos de acoplamento, são apresentados quatro tipos de acopladores: Capacitivos, Indutivos, Resistivos e do tipo Antena. Os acopladores resistivos são indicados para aplicações em BT e os acopladores do tipo antena apresentam significativos níveis de atenuação, portanto são desconsiderados neste estudo. Os acopladores capacitivos são constituídos por um conjunto de capacitores conectados em série, de modo a prover o nível de tensão que suporte a tensão nominal do sistema em que o mesmo será conectado [3]. Contudo, em função da necessidade de abertura do circuito de MT (as subestações do sistema reticulado utilizam terminal desconectável reto, sem derivação) para instalação do acoplador, inviabiliza sua utilização. 
Os acopladores indutivos em geral são do tipo "Split Core" (isolado). Não exigem o desligamento do sistema elétrico para sua instalação, sendo assim, mais simples e de menor complexidade. O formato toroidal do núcleo minimiza os fluxos de dispersão e o material policristalino apresenta elevada permeabilidade magnética em faixa de frequência correspondente a das tecnologias PLC que seguem o padrão IEEE 1901.2a (até $500 \mathrm{kHz}$ ) [6]. Possuem um custo relativamente elevado quando comparado ao restante do sistema, além disso, há a necessidade, como no caso dessa pesquisa, que o acoplador seja resistente a alagamentos de subestações subterrâneas. Além disso, é necessário frisar que a totalidade dos acopladores existentes no mercado foram desenvolvidos para a utilização em redes de MT que não correspondem às características da rede brasileira. Desta forma, faz-se necessário averiguar a possibilidade da utilização destes dispositivos nessa rede elétrica.

\section{Estudo de caso}

$\mathrm{O}$ estudo de caso utilizado neste artigo refere-se a um projeto de $\mathrm{P} \& \mathrm{D}$ que tem por objetivo o desenvolvimento de um sistema de comunicação PLC, que permite a interligação de um conjunto de 160 subestações subterrâneas de energia elétrica, que compõem o sistema reticulado de distribuição de energia localizado no centro da cidade de Porto Alegre, pertencente a concessionária CEEE-D. O acoplador utilizado nos testes, é fabricado pela empresa PREMO (Espanha), modelo MICU 300A $\mathrm{OH} / \mathrm{LF}$, com corrente nominal de $300 \mathrm{~A}$, tensão de trabalho de até $24 \mathrm{kV}$, faixa de frequência de operação de $30 \mathrm{kHz}$ a $500 \mathrm{kHz}$ (cobrindo toda a faixa de frequência de NB PLC regido pelo padrão IEEE 1901.2a) [6].

Seguindo as linhas de pesquisa já discutidas duas estratégias são avaliadas para projeto de comunicação utilizando o sistema PLC: (i) estratégia baseada em acoplador e (ii) estratégia baseada no uso dos transformadores de distribuição do sistema reticulado como elementos de acoplamento. No caso da primeira estratégia, que é baseada nos transformadores como elementos de acoplamento, faz-se necessário conhecer o comportamento desses dispositivos em relação a sua resposta em frequência na faixa de frequência dos modems PLC com potencial uso na solução final. Contudo, dado ao limite de páginas do artigo, serão apresentados os ensaios utilizando um par de modens PLC PL360, configurados no padrão G3 e modulação DBPSK_ROBO (modo robusto, onde há envio repetido de símbolos), transmitindo pacote de dados de 97 bytes, conforme descrito a seguir:

1. Aplicação de sinal PLC a um enrolamento secundário (com relação ao neutro) do transformador de distribuição. Os demais terminais do secundário, conectados ao neutro por uma resistência de 50 ohms. A leitura do sinal é realizada em um terminal secundário (com relação ao neutro) do transformador de distribuição, com os demais terminais do secundário conectados ao neutro por uma resistência de 50 ohms (o terminal de injeção de sinal e de leitura foi o X1);

2. Aplicação de sinal PLC a um enrolamento secundário (com relação ao neutro) do transformador de distribuição. Os demais terminais do secundário são conectados ao neutro por uma resistência de 50 ohms e a leitura de sinal usando acoplador indutivo e Analisador de Espectro em um terminal primário;

3. Aplicação de sinal PLC a um enrolamento primário do transformador de distribuição usando acoplador indutivo. A leitura do sinal, é realizada usando acoplador indutivo e Analisador de Espectro em um terminal primário diferente do transformador de distribuição, com os terminais do secundário conectados ao terra por uma resistência de 50 ohms.

Em todos os ensaios descritos anteriormente os transformadores não estavam energizados. Foram ensaiados transformadores de $75 \mathrm{kVA}, 150 \mathrm{kVA}$ e $225 \mathrm{kVA}$ de uso em redes aéreas e um 
transformador de $500 \mathrm{kVA}$ de uso em sistemas subterrâneos (mesmo modelo usado no sistema reticulado da CEEE-D no centro da cidade de Porto Alegre). Em relação aos ensaios descritos nos itens anteriores, em todos os ensaios os 1000 pacotes de dados foram recebidos sem erro. Nesse aspecto o desempenho é idêntico. Contudo, ao observar o parâmetro RSSI coletado pelo módulo de comunicação do modem PL 360 são observadas diferenças, que são sumarizadas na Tabela 1, onde em função do limite de páginas do artigo, serão apresentados os dados realizados com o transformador de $500 \mathrm{kVA}$.

Tabela 1: Intensidade de sinal PLC recebido x Percurso de propagação do sinal. Fonte: Autor.

\begin{tabular}{cc}
\hline Percurso de propagação & RSSI \\
\hline Injeção X3 - Leitura acoplador H1 & 62 \\
Injeção X3 - Leitura acoplador H3 & 71 \\
Injeção X3 - Leitura acoplador H2 & 73 \\
Injeção X2 - Leitura acoplador H2 & 72 \\
Injeção X2 - Leitura acoplador H3 & 52 \\
Injeção X2 - Leitura acoplador H1 & 74 \\
Injeção X1 - Leitura acoplador H1 & 76 \\
Injeção X1 - Leitura acoplador H2 & 52 \\
Injeção X1 - Leitura acoplador H3 & 76 \\
Injeção acoplador H1 - Leitura acoplador H1 & 111 \\
Injeção acoplador H2 - Leitura acoplador H1 & 103 \\
Injeção acoplador H2 - Leitura acoplador H2 & 112 \\
Injeção acoplador H3 - Leitura acoplador H2 & 107 \\
Injeção acoplador H3 - Leitura acoplador H3 & 112 \\
Injeção acoplador H1 - Leitura acoplador H3 & 101 \\
Injeção X1 do transf. 1 - Leitura X1 do transf. 2 & 89 \\
\hline
\end{tabular}

Analisando os dados presentes na Tabela 1 percebe-se que o nível de perda de sinal com o uso dos acopladores é relativamente menor que os dos demais percursos de propagação. O percurso onde o sinal é injetado e coletado no secundário dos transformadores se coloca em uma posição intermediária entre as opções analisadas, apresentando uma atenuação total maior que a observada com o uso exclusivo dos acopladores indutivos, mas inferior à opção em que a injeção é realizada através do secundário do transformador e a leitura a partir de um acoplador indutivo. Contudo, conforme apresentado em [4], o transformador tem influência no comportamento do PLC apresentando característica de carga com impedância variável, quando utilizado o acoplador com elemento de conexão à rede. Nesse sentido, a modelagem matemática que descreve a impedância do transformador, observada do ponto de aplicação do sinal PLC entre o neutro e fase no primário é representada pela equação (1), dada por:

$$
Z_{L G}=\frac{3 Z_{1}\left(Z_{2}+Z_{L V}\right) Z_{3}+Z_{1} Z_{3}^{2}+\left(Z_{2}+Z_{L V}\right) Z_{3}^{2}}{3 Z_{1}\left(Z_{2}+Z_{L V}\right) Z_{3}+Z_{1} Z_{3}+3\left(Z_{2}+Z_{L V}\right) Z_{3}} .
$$

Por sua vez, a impedância observada entre fases do primário pode ser obtida através da equação (2), ou seja:

$$
Z_{L L}=\frac{2 Z_{1}\left(Z_{2}+Z_{L V}\right) Z_{3}}{Z_{1} Z_{3}+\left(Z_{2}+Z_{L V}\right) Z_{3}+\left(Z_{2}+Z_{L V}\right) Z_{3}}
$$

onde: $Z_{L V}$ representa a carga do lado de $\mathrm{BT}, Z_{1}, Z_{2}$, e $Z_{3}$ representam as impedâncias determinadas por meio das características internas do transformador. Assim, a partir dos dados 
adquiridos é possível estimar esses valores para o estudo proposto nesse trabalho, onde obteve-se $Z_{1}=0,0039+j 0,6283 \Omega, Z_{1}=10+j 0,0628 \Omega, Z_{3}=5,6549 \cdot 10^{-10} \Omega$ e $Z_{L V}=50 \Omega$.

A partir dos parâmetros estimados, foi possível avaliar a resposta do modelo em função dos dados reais do comportamento do transformador, quando aplicados sinais de alta frequência de comunicação PLC. Na Figura 3, portanto é apresentado o resultado do modelo implementado, e dos dados obtidos nos testes experimentais realizados.

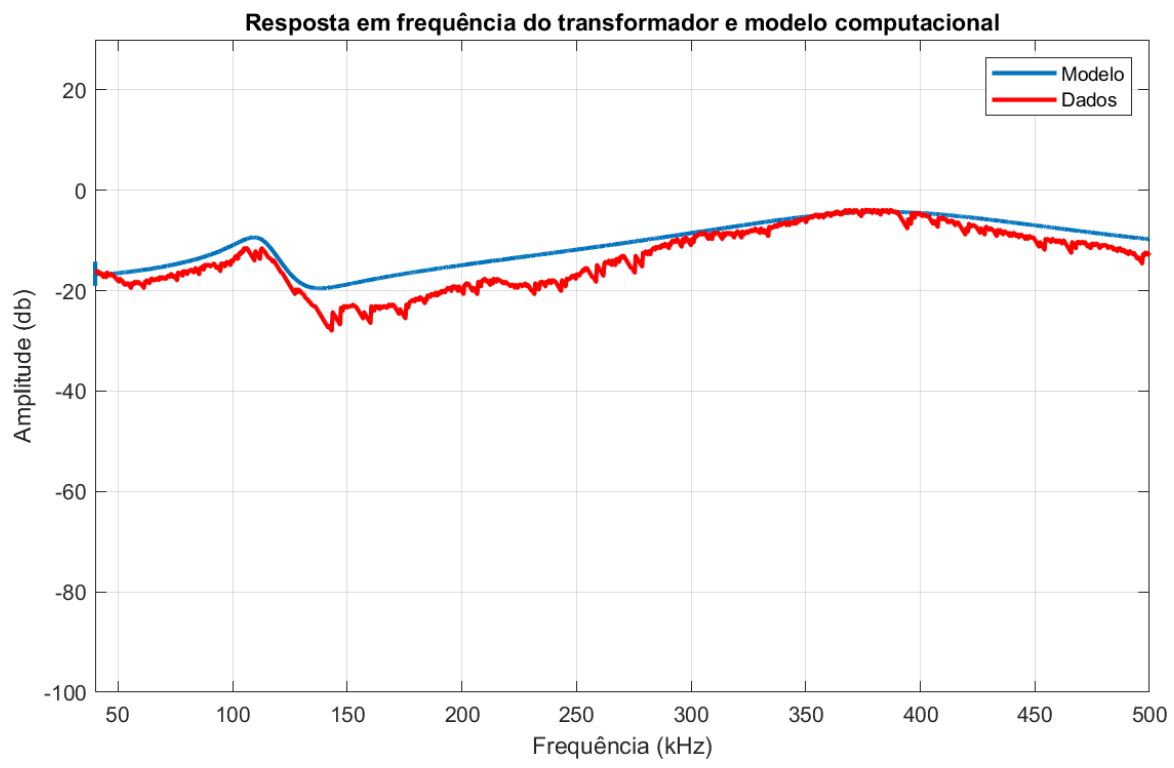

Figura 1: Resultado do modelo computacional implementado. Fonte: Autor.

Verifica-se que para toda a faixa de frequência de operação do PLC, (40 kHz à $500 \mathrm{kHz})$ há uma forte atenuação o que condiz com os dados apresentados pela Tabela 1. Contudo, para a implementação do modelo computacional é necessário levar em consideração as características do transformador para determinar as melhores características possíveis na implementação do PLC em MT por meio do acoplador indutivo.

\section{Conclusões}

Nesse artigo foi proposto o desenvolvimento de uma solução de comunicação PLC para ser aplicada no circuito de MT do sistema reticulado de distribuição de energia, localizado no centro da cidade de Porto Alegre, no RS. Inicialmente, foi realizada uma análise de formas de injeção e leitura de sinais PLC no circuito de MT, com foco no uso de dispositivos acopladores, bem como no uso dos próprios transformadores de distribuição para essa finalidade. O sucesso de uma ou de outra estratégia está relacionado à eficiência com que o sinal é injetado, de modo a se sobrepor aos ruídos presentes na rede e que são propagados dos circuitos de BT para MT. Na sequência, foi desenvolvido um modelo computacional de forma a permitir avaliar esse comportamento. Por fim, foi possível simular o comportamento de ruídos provenientes do lado da rede de BT, sem a necessidade de testes práticos. O modelo computacional implementado convergiu de forma satisfatória, quando comparado aos testes experimentais realizados em campo. No entanto, como trabalhos futuros para uma completa validação do modelo, mais estudos precisam ser efetivados, com o sistema energizado. 


\section{Agradecimentos}

Os autores agradecem ao programa de $\mathrm{P} \& \mathrm{D}$ Aneel e à CEEE-D pelo financiamento do projeto "Interligação de Subestações de Sistema Reticulado Subterrâneo através de Rede de Comunicação PLC (Power Line Communication) operando sobre o barramento de Média Tensão", objeto do contrato CONVEX N ${ }^{o}$ CEEE-D/ 5000002033.

\section{Referências}

[1] Ahmad, A. Contribution à la modélisation des transformateurs de puissance et de leur comportement en haute fréquence, Thesis, Ecole Centrale de Lyon, 1992.

[2] Aljohani O., Abu-Siada A. and Shengtao L. High frequency power transformer modelling for frequency response analysis studies, International Conference on Condition Monitoring and Diagnosis (CMD), 5:291-294, 2016. DOI: 10.1109/CMD.2016.7757818.

[3] Costa, L. G., Queiroz, A., Adebisi, B., Costa, V. and Ribeiro, M. Coupling for Power Line Communications: A Survey. Journal of Communication and Information Systems, 32:8-22, 2017. DOI: doi.org/10.14209/jcis.2017.2.

[4] Franek L. and Fiedler P. A Multiconductor Model of Power Line Communication in MediumVoltage Lines, Energies, 10:816, 2017. DOI: 10.3390/en10060816.

[5] Gustavsen B. Eliminating Measurement Cable Effects From Transformer Admittance Measurements, IEEE Transactions on Power Delivery, 31:609-1617, 2016. DOI: 10.1109/TPWRD.2016.2536704.

[6] IEEE C. S. Ieee standard for low-frequency (less than $500 \mathrm{khz}$ ) narrowband power line communications for smart grid applications. IEEE Std 1901.2-2013, 2013, 1-269. DOI: 10.1109/IEEESTD.2013.6679210.

[7] Jurisic B., Uglesic I., Xemard A. and Paladian F. Difficulties in high frequency transformer modeling, Electric Power Systems Research, 138:25-32, 2016. DOI: 10.1016/j.epsr.2016.02.009.

[8] Kikkert, C. J. MV to LV transformer PLC bypass coupling networks for a low cost smart grid rollout, IEEE PES Innovative Smart Grid Technologies, 1:1-6, 2011. DOI: 10.1109/ISGTAsia.2011.6167075.

[9] Li, X., Ge, Y. Y., Mao, X., Xue, W. and Xu, N. Improved Energy Structure Prediction Model Based on Energy Demand Forecast, IEEE Conference on Energy Internet and Energy System Integration, 1-5, 2018. DOI: 10.1109/EI2.2018.8582170.

[10] Pirak, C., Sangsuwan, T. and Buayairaksa, S. Recent advances in communication technologies for smart grid application: A review. International Electrical Engineering Congress 1:1-4, 2014. DOI: 10.1109/iEECON.2014.6925952.

[11] Sausen P., Campos M., Sausen A., Sauthier L.F., Oliveira A. C. and Oliveira S. C. Development Of A Complete System Bidirectional For Smart Metering Applications In Smart Grids, Revista Eletrônica de Potência, 21:148-157, 2016. DOI: 10.18618/REP.2016.2.2619.

[12] Sauthier L. F., Oliveira A.C., Campos M., Sausen P., Sausen A. e Luciano B. Estratégias de acoplamento de sinais Power Line Communication em circuitos de média tensão, Anais do Congresso Brasileiro de Automática 2020, volume 2, 2020. DOI: 10.48011/asba.v2i1.1307. 